\title{
Squeezing Uncertainty from Saccadic Compression
}

\author{
C. S. Chapman \\ University of British Columbia
}

\author{
A. R. Hunt \\ University of British Columbia
}

\author{
A. Kingstone \\ University of British Columbia
}

\begin{abstract}
Brief visual stimuli presented before and during a saccade are often mislocalized due to spatial compression. This saccadic compression effect is thought to have a perceptual basis, and results in visual objects being squeezed together and their number underestimated. Here we show that observers are also uncertain about their visual experiences just before and during a saccade. It is known that responses tend to be biased away from extreme values under conditions of uncertainty. Thus, a plausible alternative explanation of compression is that it reflects the uncertainty-bias to underestimate the number of items that were presented. We test this hypothesis and find that saccadic compression is independent of certainty, and is significantly modulated by orientation, with larger effects for stimuli oriented horizontally, in the direction of the saccade. These findings confirm that saccadic compression is a perceptual phenomenon that may enable seamless perceptual continuity across saccades.
\end{abstract}

\section{Keywords: \\ Eye Movements, Receptive Fields, Saccadic Compression, Decision Uncertainty}

\section{Introduction}

An important but unsolved problem in perception is how we maintain a seamless perception of the visual world despite the global shift in retinotopic positions that occurs every time the eyes move. The subjective continuity of our visual experience across saccades implies that information is not processed normally when they occur. One key to understanding how we maintain this continuity is the systematic mislocalization of stimuli in both space (e.g. Honda, 1989; Matin, \& Pearce, 1965; Schlag \& Schlag-Rey, 1995; review Ross, Morrone, Goldberg \& Burr, 2001) and time (Morrone, Ross \& Burr, 2005; Yarrow, Haggard, Heal, Brown, \& Rothwell, 2001; but see Hunt, Chapman \& Kingstone, 2007) around the time of a saccade. Matin and colleagues were among the first to demonstrate the spatial mislocalization of targets presented during (Matin, \& Pearce, 1965; Matin, Matin \& Pearce, 1969) and immediately before (Matin, Matin \& Pola; 1970) a saccade. Later work demonstrated that saccadic mislocalization was dependant not only on the time of stimulus presentation within a saccade (Honda, 1989), but also on the availability of background visual cues and the physical position of the stimulus (Honda, 1993).
Honda (1991) also showed that mislocalization happened for vertical saccades, and later noted that localization errors were only ever present in the direction of the saccade, and not orthogonal to it (Honda, 1993). More recently, Ross, Morrone \& Burr (1997) have shown a precise pattern of saccadic compression, where the perceived locations of visual stimuli are compressed towards the goal of the saccade (see also Morrone, Ross, \& Burr, 1997).

In the original saccadic compression experiment, visual stimuli flashed immediately before and during the saccade were systematically mislocalized towards the goal of the saccade (Ross, Morrone, \& Burr, 1997). In an extreme example of this phenomenon (Morrone, Ross, \& Burr, 1997), multiple items were "compressed" together, resulting in a systematic underestimation of the number of items when they were displayed immediately before a saccade was executed. This compression of perceived space around the time of a saccade was thought to reflect processes that compensate for eye movements to support a stable perception of the visual world. 
A plausible alternative explanation for saccadic compression exists, however. Research in other areas of psychophysics has demonstrated that judgments based on memories of perceptual events tend to systematically regress towards the mean, with more extreme responses being under-represented (e.g., McNamara \& LeSueur, 1989). This memory-compression effect is thought to occur either because of increased uncertainty about the stimulus display, leading to a response biases away from extreme values (Kerst and Howard, 1985), or because of a loss of information in the encoding and retrieval process, resulting in underestimations (Radvansky, CarlsonRadvansky, \& Irwin, 1995). Regardless of the mechanism underlying it, the existence of the memory-compression effect raises the concern that the compression effect in saccade experiments could be due not to perceived spatial distortions, but to increased uncertainty about remembered perceptual events that occurred around the time of a saccade. That is, when subjects are uncertain about the location of an event because it occurred around the time of a saccade, their localization responses may be biased toward the location they are currently fixating, resulting in compression-like effects. Similarly, when subjects must report the number of items appearing on the screen, they would be biased away from reporting larger numbers when uncertain.

In the present experiment we examined the effect of certainty on the compression effect. We adapted the multiple items paradigm of Morrone, Ross and Burr (1997), presenting up to four squares on the screen in each saccade trial and asking subjects both how many squares they saw and whether or not they were sure of their response. A significantly larger compression effect under conditions of response uncertainty would suggest that compression is due, at least in part, to systematic guessing strategies. As an additional test of the uncertainty hypothesis in the present experiment, multiple squares were always aligned horizontally, vertically or diagonally. Previous work has shown that compression as observed in localization errors is smaller in the direction orthogonal to the saccade than in the same direction (Honda, 1993; Kaiser and Lappe, 2004). Therefore, in our experiment, one would expect a genuine loss due to compression to be greatest for the horizontal and diagonal orientations, as these both contain a spatial component in the direction of the eye movement.

\section{Methods}

We measured saccadic compression in ten naive observers, who completed at least 480 trials each. Each subject placed their chin in a chin-rest $38 \mathrm{~cm}$ away from a 17 in CRT monitor $(80 \mathrm{~Hz}, 32.5 \times 28 \mathrm{~cm})$, such that the monitor subtended $46.3^{\circ} \times 40.4^{\circ}$. We presented from 0 to 4 black squares $\left(3.4^{\circ}\right)$ for $12.5 \mathrm{~ms}$ (one monitor refresh) on a white background before, during, or after a $36.5^{\circ}$ rightward saccade from one fixation point to another. Observers were asked to enumerate how many objects they perceived by entering a number from 0 to 4 . They were then prompted to reply yes (by pressing 'y') or no (by pressing ' $n$ ') to the question "are you sure?", which was presented at the center of the screen (see Figure 1 for an illustration of the sequence of events in a trial).

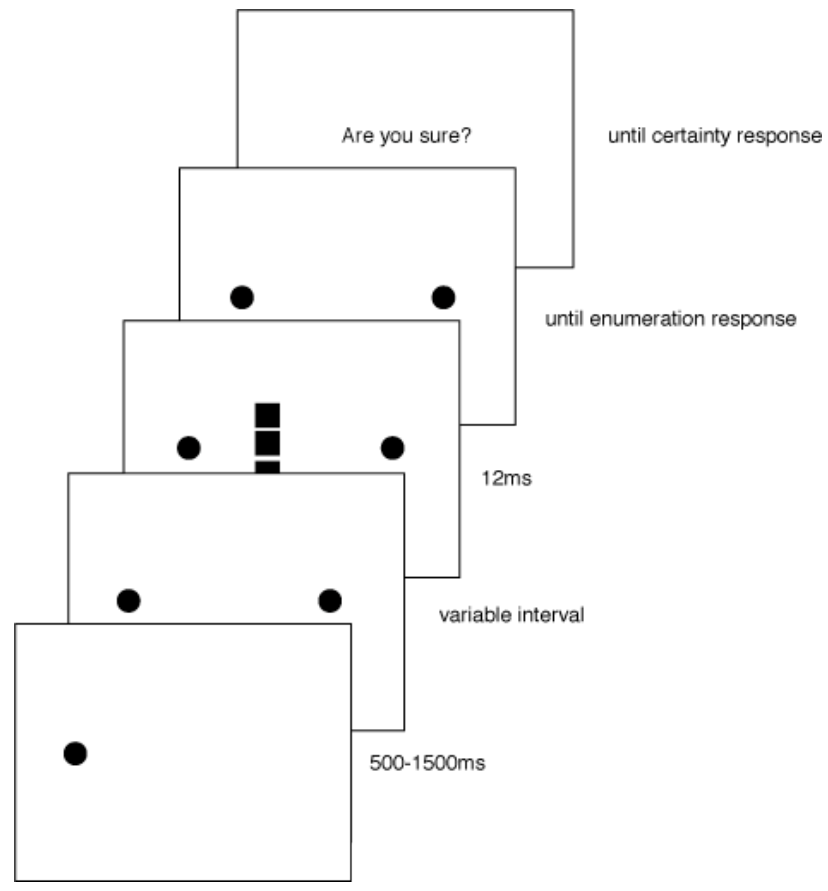

Figure 1. Series of events in a typical trial in the current study. Each trial began with a fixation dot on the left side of the screen. After a random interval between 500-1500ms, a second dot appeared on the right side of the screen and a beep sounded to signal the observer to execute a saccade. After a variable interval based on a running mean of the observer's saccade reaction time, 0-4 squares appeared for $12 \mathrm{~ms}$. Each square appeared in one of 41 possible locations, and multiple squares were always aligned horizontally, diagonally, or vertically. The fixation points remained on the screen until observers responded about the number of items they thought had been presented (0 to 4). This response brought up a prompt asking if they were sure (yes or no). The response to the question of certainty started the next trial. 
The $3.4^{\circ}$ squares were horizontally, vertically, or diagonally aligned, with $7.7^{\circ}$ between the centers of each horizontally or vertically aligned square. The array of possible locations of the squares consisted of an imaginary $5 \times 5$ square grid and an imaginary $4 \times 4$ diamond grid, superimposed in the center of the display. This configuration ensured that each orientation condition was equated in terms of the number of possible spatial positions. The total width of the array of locations was $34.2^{\circ}$. The number of squares was also randomized, with 0 squares presented on $12.5 \%$ of the trials, 1 square presented on $12.5 \%$ of the trials, and 2,3 , or 4 squares each presented on $25 \%$ of the remaining trials.

The stimuli were presented at a variety of intervals before, during, and after the expected time of the saccade. To achieve this, the saccadic onset latency was collected on-line, using an EyeLink II, from individual subjects, and the running average saccade latency was used to predict the onset time of subsequent saccades. Because we were most interested in the time period immediately prior to a saccade, $50 \%$ of trials occurred $50 \mathrm{~ms}$ prior to average saccade latency, 25\% occurred during the saccade (display triggered by saccade onset), $12.5 \%$ occurred $100 \mathrm{~ms}$ before the average saccade latency, and $12.5 \%$ occurred $200 \mathrm{~ms}$ after average saccade latency. Trials were re-coded offline into four critical time periods according to when the stimuli were actually presented relative to the onset of the saccade based on the timing of the onset of the stimuli: $>100 \mathrm{~ms}$ before a saccade, $<100 \mathrm{~ms}$ before a saccade, during a saccade (peri), or after a saccade (post).

\section{Results}

Figure 2a shows the effect of the saccade on certainty. Observers are the most uncertain about their responses around the time of an eye movement, reinforcing the concern that compression is brought on by an increase in uncertainty that occurs around the time of an eye movement, rather than the eye movement per se. To calculate an index of compression we took the mean of the difference between the actual number of squares presented on a given trial and the participants' report of the number of squares perceived. Figure $2 \mathrm{~b}$ shows the overall compression effect across the four time periods. We replicated the critical finding that compression occurs significantly more for stimuli presented during and immediately prior to a saccade relative to well before $(>100 \mathrm{~ms}$ prior) or after the saccade ${ }^{1}$. To assess the role of uncertainty in the compression effect, we examined compression as a function of both certainty and time period using a repeatedmeasures analysis of variance.
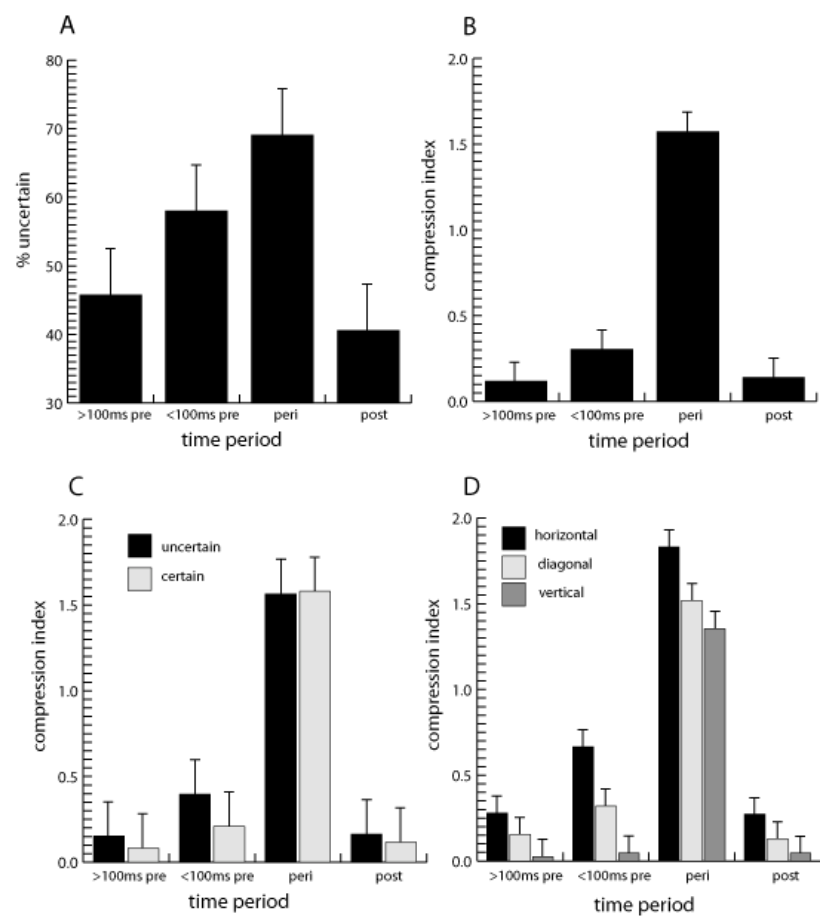

Figure 2: A. The percent of uncertain responses for judging stimuli appearing at different time points relative to the onset of the saccade. Note that uncertainty is largest immediately prior to and during a saccade. B. Compression (calculated as a mean of the perceived number minus actual number of squares) across the time periods. Note that compression is also largest immediately prior to and during a saccade. C. Compression as a function of both certainty and time period. Compression is influenced by time period, but not by certainty, and there is no interaction of certainty with time. D. The effect of orientation on compression is shown for each time period. The orientation effect interacts with time period, with the larger orientation effects immediately before and during the saccade.

\footnotetext{
${ }^{1}$ The larger compression effect in the perisaccadic relative to the presaccadic case is consistent with the previous studies (e.g. Morrone et al., 1997; Kaiser \& Lappe, 2004). Distortions of space begin 50ms before the eye movement, and tend to peak around, or shortly after, the onset of the saccade. The presaccadic category in our study therefore includes instances where spatial distortions would be small, or not yet have begun, while the perisaccadic category contains a large proportion of trials for which compression is expected to be at its peak.
} 
There was a strong effect of time period (that is, when the stimuli were displayed relative to the onset of the eye movement) $[\mathrm{F}(3,27)=87.23, \mathrm{p}<.001]$, but there was no effect of certainty on compression $[F(1,9)=1.74]$ and no interaction of certainty with time period $[\mathrm{F}(3,27)<1]$. This result, shown in Figure 2c, implies that the compression effect is independent of the reported certainty of the observer. Finally, Figure $2 \mathrm{~d}$ shows the effect of orientation on compression. There was a significant effect of orientation $[\mathrm{F}(2,18)=41.63, \mathrm{p}<.001]$, with strongest compression in the direction of the saccade (horizontal), and weakest orthogonal to the saccade (vertical). There was also a significant interaction of orientation with time period $[\mathrm{F}(6,54)=4.22, \mathrm{p}<.01]$. As can be seen from Figure $2 \mathrm{~d}$, this interaction occurred because the effect of orientation was largest immediately before and during the saccade, indicating it is significantly modulated by genuine compression.

\section{Discussion}

We set out to explore the memory-compression effect as a plausible alternative explanation for the saccadic compression effect. We conclude that the saccadic compression effect is a perceptual phenomenon, and not the result of memory or response-related effects. Two of the present findings support this conclusion. First, if the memory-compression effect were the basis for compression, compression would be significantly larger when subjects were uncertain about their responses. Instead, we found that the significant changes in response certainty around the time of a saccade did not impact the occurrence of presaccadic compression. The fact that the compression effect was not significantly reduced in magnitude, even when observers were sure of what they saw, supports the theory that presaccadic compression is a perceptual phenomenon. Second, the memorycompression hypothesis also predicts no relationship between the orientation of the stimulus and the amount of compression. We observed that stimulus orientation modulates the compression effect, such that stimuli with an orientation component in the direction of the saccade show larger compression. This orientation effect was modulated by time period, with larger orientation effects for stimuli displayed immediately prior to and during a saccade. Together these results suggest that perceptual maps of space are literally compressed toward the goal, and in the direction of a saccade immediately before and during its execution.
A saccade-induced remapping of receptive fields to be centered on the coordinates of the target of an impending saccade could facilitate a seamless perception of space across eye movements. Evidence of a pathway that conveys information about oculomotor commands from the superior colliculus of the midbrain up to the cortical frontal eye fields (Sommer and Wurtz, 2006) provides physiological evidence that retinotopically-organized cortical maps can anticipate and compensate for changes in eye position as they occur. Saccadic compression may reflect a functionally important consequence of this remapping process. Based on the present findings, we are confident that further research into this phenomenon will bear new insights into how our seamless perception of space is maintained in the face of the constant shift of spatial locations that accompanies saccadic eye movements.

\section{References}

Honda, H. (1989). Perceptual localization of visual stimuli flashed during saccades. Perception and Psychophysics, 45, 162-174.

Honda, H. (1991). The time courses of visual mislocalization and of extra-retinal eye position signals at the time of vertical saccades. Vision Research, 31,19151921.

Honda, H. (1993). Saccade-contingent displacement of the apparent position of visual stimuli flashed on a dimly illuminated structured background. Vision Research, 33, 709-716.

Hunt, A.H., Chapman, C.S. \& Kingstone, A. (in press). Taking a long look at action and time perception. Journal of Experimental Psychology: Human Perception and Performance.

Kaiser, M. \& Lappe, M. (2004). Perisaccadic mislocalization orthogonal to saccade direction. Neuron, 41, 293300 .

Kerst, S.M. \& Howard, J.H. (1978). Memory psychophysics for visual area and length. Memory and Cognition, 6, 327-325.

Matin, L., Matin, E. \& Pearce DG. (1969). Visual perception of direction when voluntary saccades occur: I. Relation of visual direction of a fixation target extinguished before a saccade to a subsequent test flash presented during the saccade. Perception and Psychophysics, 5,65-80. 
Matin, L., Matin, E. \& Pola, J. (1970). Visual perception of direction when voluntary saccades occur: II. Relation of visual direction of a fixation target extinguished before a saccade to a subsequent test flash presented before the saccade. Perception and Psychophysics, 8,9-14.

Matin, L. \& Pearce, D.G. (1965). Visual perception of direction for stimuli flashed during voluntary saccadic eye movements. Science, 148,1485-1488.

McNamara, T. \& LeSueur, L.L. (1989). Mental representation of spatial and nonspatial relations. Quarterly Journal of Experimental Psychologgy, 41A, 215-233.

Morrone, C.M., Ross, J., \& Burr, D. (1997). Apparent position of visual targets during real and simulated saccadic eye movements. The Journal of Neuroscience, 17, 7941-7953.

Morrone, M.C., Ross, J. \& Burr, D. (2005). Saccadic eye movements cause compression of time as well as space. Nature Neuroscience, 8,950-954.

Radvansky, G.A., Carlson-Radvansky, L.A. \& Irwin, D.E. (1995). Uncertainty in estimating distances from memory. Memory \& Cognition, 23, 596-606.

Ross, J., Morrone, C.M. and Burr, D. (1997). Compression of visual space before saccades. Nature, 386, 598-601.

Ross, J., Morrone, M.C., Goldberg, M.E. \& Burr, D.C. (2001). Changes in visual perception at the time of saccades. Trends in Neurosciences, 24(2):113-21.

Schlag, J. \& Schlag-Rey, M. (1995). Illusory localization of stimuli flashed in the dark before saccades. Vision Research, 35,2347-2357.

Sommer, M.A. \& Wurtz, R.H. (2006). Influence of the thalamus on spatial visual processing in frontal cortex. Nature, 444, 374-377. 